letzteren gelegentlich folgende vermerkt: S. $522{ }^{27}$ muss es heissen: D. I 12, 1 \$ ; S. $522^{28}$ : C. Th. XVI 4, 5; S. $523^{30}$ : Iul. im C. Th. XII; S. $531^{\circ}$ : D. XXVI 7; S. $533^{10}$ : Phil, im G. Iust. IV 19; S. $534^{18}$ Z. 31 : D. XXXII, 1. 29; $535^{19}$ Z. 13: D. II 14, 1. 47; S. $53 \tilde{5}^{18}$ Z. 14 : D. XXXII, 1. 91; S. $538^{28}$ Z. 4: D. XXVI 7; S. $538^{20}$ Schluss: D. III 5, 1. 33; S. $554^{\text {в }}$ Z. 2: D. II 14.

Halle.

Th. Niemeyer.

Jacques Flach, Etudes critiques sur l'histoire du droit romain au moyen âge avec textes inédits. Paris, L. Larose et Forcel, 1890. 336 S., $8^{0}$.

Unter diesem Titel hat der Verfasser, Nachfolger Laboulayes auf dem Lehrstuhl für vergleichende Rechtsgeschichte an dem Collège de France, drei Abhandlungen vereinigt und seinem Vorgänger gewidmet. Dieselben beziehen sich sämmtlich auf die mittelalterlichen Schicksale des römischen Rechts; während aber die beiden letzten nur einzelne Sonderpunkte behandeln, hringt die erste die ganze viel unstrittene Frage nach einer frühmittelalterlich-romanistischen Wissenschaft zur Erörterung und erscheint demgemäss als die weitaus wichtigste. Beigegeben ist jedem Hauptabschnitte (S. 129-183 und S. 225-286) ein reiches und ausserordentlich schätzbares Material von Veröffentlichungen aus den handschriftlichen Schätzen der Pariser Nationalbibliothek: Glossen, Zusammenstellungen von Anfangsworten, synoptische Tabellen zu Petrus - Manuscripten u. s. f.: für das Einzelne muss hier, Mangels des nöthigen Raumes, auf das Buch selbst verwiesen werden; für die Zuverlässigkeit und Akribie der Ausgahe bürgt die hekanıte aussergewöhnliche Sachkunde, dipiomatische Schulung und Genauigkeit des Verfassers.

Die erste Abhandlung besteht in einer kühlen, besonnenen, in kurzen Worten oft viel sagenden, oft aber doch auch etwas aphoristischen Nachprüfung der (wesentlich Fittingschen) Beweise für die Behauptung, dass durch alle, selbst die dunkelsten Zeiten des Mittelalters hindurch, stets Wissenschaft, Litteratur und Unterricht des römischen Rechts Platz gegriffen habe. Sympathisch wie diese Behauptung unserem evolutionistischen Zeitalter klingt (S. 13 f.), wird sie doch nach Flach durch die Thatsachen wenig gerechtfertigt; zu den mannigfachen Angriffen, welche Fittings Hypothesen und Darstellung letzthin erfahren mussten, gesellt sich somit die Flachsclse Untersuchung hinzu. Wenn dieselbe auch nicht viel neue Gegenargumente bringt, sondern sich namentlich durch ein fortwährendes non liquet auszeichnet, so ist sie doch positiv in der Negation der Fittingschen Continuitätstheorie; sie beruht auf dem von Conrat in seiner Einleitung zu der Ausgabe der Epitome Exactis regibus begonnenen Angriff und leite zu dem umfassenden Angriff über, zu welchem Conrat in seiner grossen, augenblicklich im Gange befindlichen Veröffentlichung ${ }^{1}$ ) vorgeht.

2) Mit der positiven Bedeutung eines umfassenden Quellen-Werkes, 
Flach zerlegt den Stoff in drei Unter-Abtheilungen: 1) Wie steht es um diejenigen Schriften, welche wohl nicht zwischen Ende des 6 . und des 11. Jahrhunderts, sondern entweder nachher oder vorher entstanden sind? Welchen Werth hat namentlich, falls letzteres der Fall, der Umstand ihrer Ueberlieferung durch diese dunklen Jahrhunderte hindurch? 2) Welches ist die Bedeutung der uns wirklich in Manuscripten aus der Zeit zwischen Ende des 6. und des 11. Jahrhunderts überlieferten Arbeiten? 3) Wie steht es während dieser kritischen Zeit um den Rechtsunterricht? - An die Behandlung der ersten Frage werden Bemerkungen über die Benutzung und das Vorkommen von Citaten römischer Rechtsquellen während des fraglichen Zeitraumes angeschlossen.

Merkwürdiger Weise zählt Flach zu der ersten Gruppe nur solche Werke, welche Fitting in seinen ,Juristischen Schriften des früheren Mittelalters" veröffentlicht hat: Actionentractate, juristische Vocabularien, Fragmente von Institutionencommentaren, Compendium iuris, De iustitia. Von allen diesen nimmt Flach entweder an, und zwar dies mit Vorliebe, dass sie ihre Entstehung erst dem Beyinn wissenschaftlicher Regungen um das Ende des 11. Jahrhunderts, sei es den unmittelbaren Vorläufern der Bononiensischen Schule, sei es den ersten Anregungen dieser schon erblühten Schule nach auswärts, verdanken; oder dass sie vor dem Ende des 6. Jahrhunderts im wesentlichen entstanden, durch die dunkeln Jahrhunderte aber in einer solchen Art und Weise überliefert worden sind, welche keinerlei Spur wissenschaftlichen Verständnisses oder theoretischer Pflege erkennen Jassen. Welche Alternative für jede einzelne Schrift zutreffe, entscheidet Flach regelmässig nicht; dem neu entdeckten Juristen Geminianus ist er wenig hold und möchte, unter Benutzung der Resultate von Pescatores Irnerius-Forschung, unter dem G., qui primus tractavit de natura actionum, eben Irnerius verstehen.

Mehr Bedeutung misst Verfasser denjenigen Schriften bei, deren vorbononiensischer Ursprung schon durch die Manuscripte feststeht. Als solche stellt er aus dem reinen rönischen Recht zusammen: 1) Institutionsglossen: Turin; Bamberg; Monte-Cassino; Paris; Köln. 2) Zum Codex: Summe von Perusia; Glossen von Pistoia und von Paris. 3) Zum Julian: Summen, Scholien und Glossen. 4) Summen und Glossen zum Theodosischen Codex und 5) zum Breviarium. Diesen Producten werden diejenigen romanistischen Darstellungen an die Seite gestellt, welche im Dienst des kanonischen, lombardischen oder Lehusrechtes stehen, einschliesslich der canonistischen Sammlungen und der

wie es Conrat, „Geschichte der Quellen und Litteratur des römischen Rechts im früberen Mittelalter" theils schon ist, theils zu werden verspricht, wollen und dürfen natürlich Flachs Etudes nicht verglichen sein; leider haben letztere ersteres nicht mehr henutzen können. Dass dabei Flach in irgend ein ${ }^{*} \mathrm{~m}$ mikroskopischen Detailpunkt, auf welchen er gerade seine ganze Aufmerksamkeit gerichtet hat, auch einmal Conrat zu verbessern in der Lage ist (S. 202), das ändert an dem Gesammtverhältniss nichts. 
Expositio ad librum Papiensem und dgl. In knappster Darstellungt werden hier mehr innerliche, auf den wissenschaftlichen Werth gerichtete Untersuchungen angestellt; das Ergebniss ist, dass alle älteren Stücke barbarisehes Gemengsel ältester Schulweisheit ohne inneres Verständniss mit lediglich grammatikalischer Bedeutung seien; während die tüchtigeren Leistungen der Zeit gegen 1070, namentlich der die Auffassung des römischen Rechts als raison écrite vorbereitenden damaligen Lombardistenschule, entstammen. Man sieht, wie wesentlich fïr Flach diese Sonnenaufgangsepoche von einigen vorbononiensischen Jahrzehnten ist, welchen er nach dem Vorgange von Conrat das zuschreibt, was man bis dahin über die vorhononiensischen Jahrhunderte vertheilt hatte.

Nach diesen Ergebnissen wird es Flach S. 104 f. leicht, dasjenige, was man als Spuren eines eigentlich wissenschaftlichen Rechtsunterrichts ausgelegt hat, als rein grammatikalische oder rhetorische Schulübungen, bedingt durch die bekannte scholastische Methode, anzusprechen: wie denn ja schon bei Quintilian das genus iudiciale als Theil der Rhetorik auftritt. Dass vor dem 12. Jahrhundert in Orléans eine Rechtsschule bestanden habe oder in Frankreich der Brachylogus entstanden sei, leugnet Flach bestimmt; "würde aber," so sagt er S. 117, ,bewiesen, dass der Brachylogus in Frankreich während des 12. Jahrluunderts entstanden ist, so würde dieser Umstand, weit entfernt davon, einen Anhaltspunkt für die Behauptung einer angenommenen Continuität des römischrechtlichen Unterrichts in unserem Lande durch das ganze Mittelalter hindurch zu geben, vielmehr bezeugen, dass diese Studien bei uns eine hervorragende Wiedergeburt in Folge und unter dem Druck der von Bologna ausgehenden Bewegung erlebt haben." - Bleiben die italienischen Schulen. Die bekannte Odofredische Stelle wird ganz wörtlich so interpretirt, dass bei Untergang des römischen Studiums von dort nicht dieses, sondern bloss die Rechtsbücher nach Ravenna übertragen wurden. Mit der längeren Existenz dieser ravennatischen Schule muss dann Verfasser rechnen, wie er ilhr den Petrus wenigstens hier nicht ausdrücklich abspricht; das hohe Lob aber, welches ihr Fitting spendet; lässt er ihr nicht zukommen, und hierin kann, ihm Berichterstatter nur zustimmen: die aequitas, welche in Ravenna gehandhaht wurde, ist nicht diejenige einer üher das strenge Recht fortgeschrittenen, des Buchstabens entbehren könnenden Epoche, sondern diejenige einer noch ungebildeten Zeit, welche mit den Quellen nicht umzugehen versteht und darum einem dunklen Rechtstriehe folgt. Wie sich dann die Besserung gegen Ende des 11. Jahrhunderts anbahnt, wird treffend hervorgehoben; ob wirklich dafür, dass schliesslich die neue Richtung statt in Ravenna vielmehr in Bologna zum Durchbruch kam, politische Gründe, Beziehungen zum Streite zwischen Papst und Kaiser massgebend waren, wie Flach vermeint, möchte ich dahingestellt sein lassen; dagegen kann ich mich ihm wieder ganz anschliessen, wenn er zu Ende den Hauptnachdruck auf die geniale Persönlichkeit des Irnerius legt (S. 126): „In Ravenna war es dem römischen Rechte nicht gelungen, sich aus den 
Banden der Praxis, Dialektik und Grammatik zu lösen. In Bologna sprengt das Genie eines Mannes seine Fesseln, es nimmt seinen Aufschwung und wird eine Wissenschaft."

Wenn freilich hiermit jede Spur einer Wissenschaft vor Bologna geleugnet werden soll, so kann man schon desshalb Verfasser nicht ganz beipflichten, weil seine Untersuchungen so vollstãndige nicht sind, dass sie zu derartig abschliessendem Urtheile berechtigen. Das weiss er selbst sehr wohl, wie er denn z. B. die ganze fundamentale Materie des Petrus und des Brachylogus principiell aus der Besprechung, als noch nicht reif zur Entscheidung, ausgeschieden hat (S. 59. 60). Aber auch sonst, fürchte ich, wird seine Polemik als eine erschöpfende fast nirgendwo bezeichnet werden können, da er fast alles unentschieden lässt und nur gerade die Fittingsche Auffassung negirt. Sobald man jedoch einen derartigen Anspruch, welchen er selbst keineswegs erhebt, fallen lässt, wird sich andererseits eine weitgehende Berechtigung seiner einzelnen Bemerkungen und Einwände nicht verkennen lassen; die Klarheit und die Zurückhaltung der Beurtheilung fallen überall vortheilhaft auf; zweifellos methodisch richtig ist die stete Rückverweisung auf die objective, handschriftliche Grundlage; bei den melır subjectiven Fragen der Werthbeurtheilungen und ähnlichen hört freilich jeder feste Massstab auf, wennschon Berichterstatter Verfassers Geschmack vielfach theilt.

Die zweite Abhandlung hängt mit der ersten insofern zusammen, als sie einen Beitrag zur Ausfüllung einer dort bemerkten Lücke, zur Kenntniss der Handschriften von Petrus Exceptiones, liefert. Sie besteht in einer mit äusserster Feinlıeil und Vorsicht, mit Scharfsinn und Geschick geführten Untersuchung über die Pariser Petrus-Manuscripte; die Einzelheiten, wennschon aut ihnen der Werth der Arbeit wesentlich beruht, können hier nicht einmal andeutungsweise berücksichtigt werden. Zunächst wird das Ergebniss festgestellt, dass die jüngst aus der Bibliothek Asburnham zu Tage gekommenen Nummern Barrois 336 und 285 zusammen, nachdem falsche Zuthaten beseitigt, Rasuren ergänzt worden sind, das von Savigny citirte, vor 1848 aus der französischen Nationalbibliothek mit zahlreichen anderen Handschriften verscliwundene MS. Bibl. nat. lat. 4719 bilden, so dass namentlich aus den (gefälschten) Anfangsworten des einen Stückes gezogene Schlussfolgerungen wegfallen (S. 202). Dies Manuscript 4719 nun aber enthält den Petrus nicht in der Ordnung unserer Drucke, sondern so, dass die erste Hälfte das sog. Tübinger Rechtsbuch darstellt - Flach nennt sie Collection A - und die zweite Hälfte den grössten Theil ${ }^{1}$ ) des übrigen Petrus - Flach nennt sie Collection B. Dagegen ist ein weiteres Pariser Manuscript, f. lat. 1730, oder sein Archetypus, aus den beiden Collectionen A und B unter Zufügung von sechs weiteren Capiteln so zusammengesetzt, dass ein Copist nach einer ihm vorgeschriebenen, elwa durch eine Tabelle der Anfangsworte ihm bezeichneten systematischen Anordnung

1) Einige - 8 - Capitel unseres Pelrus finden sich weder hier noch dort; vgl. S. 205 und S. 220 Anm. 1. 
die Stücke der beiden Sammlungen vermischt abschrieb, so dass so im wesentlichen unser Petrus entstand: aus Fehlern und Flüchtigkeiten wird dieser Vorgang überzeugend reconstruirt. Da nun auch der Text von 1730 sich vielfach als der beste und präciseste herausstellt (S. 210), so liegt die Annahme nahe, dass er die Urform derjenigen systernatischen Zusammensetzung verschiedener Sammlungen darstellt, welche wir als Petri Exceptiones kennen. In Folge dessen gewinut es aber wieder besondere Bedeutung, dass MS. Nr. 1730 im Prolog nicht hat „Odiloni“, sondern „Saxiloni"; und nicht „Valentinae“, sondern „Florentinae" civitatis; sowie dass in ihm fehlt die berühmte Bemerkung: possessionem quam in Galliae partibus appellamus honorem: denn damit schejden die wichtigsten für den französischen Ursprung des Petrus geltend gemachten Symptome aus: ein Ursprung, welcher Flach auch sonst (S. 198) nicht wahrscheinlich erscheint. Im übrigen bedarf es, so schliesst Verfasser, weiterer handschriftlicher Studien; das hier Mitgetheilte zeigt nur, wie misslich es ist, ehe diese vollständig erledigt sind, sich ein Urtheil zu bilden; namentlich wird Rūcksicht auf weitere Zersetzung des Petrus in seine Quellen und wieder dieser in ihre Elemente, als welche sich etwa Excerptensammlungen ergeben mögen, zu nehmen sein; zu diesem Behufe giebt Flach noch einige Winke und verweist namentlich auf ein Cambridger Manuscript.

Der genauen Schilderung dieses letzteren Sammelbandes, 0. 7. 40, ist die dritte Abtheilung gewidmet. Seine Schrift ist nirgendwo älter als die Mitte des 13. Jahrhunderts; von juristisch interessanten Stücken enthält er zunächst, was Conrat entgangen war, die Epitome exactis regibus mit einem längeren, durch Flach zum Abdruck gebrachlen, stark an das 4. Gapitel des dem Placentin zugeschriebenen Tractates de varietate actionum erinnernden Prolog; ferner verschiedene kleinste Bruchstücke aller Art, betreffend römisches und kanonisches Recht; eine Reihe tralaticischer Definitionen; die Distinctiones Hugonis; und endlich die Sammlung A zu den Exceptiones des Petrus (d. h. also das Tübinger Rechtsbuch), an welche sich noch einige Excerpte und Fragmente anschliessen.

Den Eindruck aus allen diesen Stıdien und Lectüren fasst Verfasser folgendermassen nicht unzutreffend schliesslich, S. 327, zusammen:

„Es scheint im 11. und 12. Jahrhundert" (- nicht auch schon in den vorhergehenden? -) ,eine juristische Masse unpersönlicher anonymer Natur umhergetrieben zu haben, welche gewissermassen im offentlichen Eigenthum stand und aus welcher jeder unbedenklich schöpfte. Dieser gemeinsame Grundstock war gehildet und genälırt aus antiken Elementen (römische Gesetze in vollständigem oder zersetzten, erkennbarem oder der Prägung beraubten Zustand, Definitionen etc.) und aus gleichzeitigen oder neuen Elernenten (Glossen, Summen, Distinctionen, Prologen etc.) Der Unterschied zwischen diesem Grundstock und der Idee, welche man sich von der "Manier" der Glossatoren gemacht, dem Typus, welchen man als denjenigen der Glossatoren festgestellt hatte (als ob diese Manier unmittelbar alles erobert und überschwemmt hätte, in Italien und ausserhalb!), hat bewirkt, dass 
Werke für vorbononiensisch genommen wurden, welche einfach jenem Grundstock entnommen waren (was namentlich von dem Petrus zutreffen mag); dieser Unterschied hat bewirkt, dass man diese Schriften als vermittelnd zwischen der Wissenschaft des 6 . und der Wiederauferstehung am Ende des 11. Jahrhunderts angesehen hat, während sie nur eine unmittelbare Folge dieser Wiederauferstehung waren."

\section{Ernst Landsberg.}

\section{Hans Paalzow, Zur Lehre von den römischen Popularklagen. Berlin 1889. $54 \mathrm{SS}$.}

Eine Neubearbeitung der Lehre von den Popularklagen musste nothwendiger Weise den epochemachenden Aufsatz von Bruns (Zeitschrift f. Rechtsgesch. Bd. 3 S. 341 ff.) zum Ausgangspunkte nehmen. In der vorliegenden Abhandlung ist dies auch durchaus geschehen, aber der Verfasser gelangt in vielen Beziehungen zu Ergebnissen, welche den von Bruns gewonnenen widersprechen. Nach einer Darlegung des Sach- und Streitstandes (I) bestimmt P. den römischen Begriff actio popularis abweichend von Bruns unter Einschluss der gesetzlichen popularen Strafklagen, aber unter Ausschluss der interdicta popularia (II). Er behandelt sodann eingehend die prätorischen actiones populares, vertheidigt entgegen Bruns deren procuratorische Natur (III) als völlig vereinbar damit, dass (regelmässig) die ganze Klagsumme an den Kläger fällt (IV), und führt aus, dass diese actiones populares, richtiger die Vorrechte der Privatinteressenten auf ihre Anstellung, in der Regel vererblich seien (V). Solche Vererblichkeit wird für die gesetzlichen popularen Strafklagen, die übrigens nur kurz berührt werden, verneint, weil bei ihnen derartige Vorrechte nicht stattfinden (VI). Als den actiones populares „verwandte Bildungen“ bespricht $P$. in Kürze die interdicta popularia zum Schutze der res publicae, Rechtsmittel, deren procuratorische Qualität ihm zweifelhaft ist, und welche er als durch ihre reipersecutorische Natur von den actiones populares in eigentlichen Sinne geschieden ansieht, ferner die Klage de collusione detegenda, die accusatio suspecti tutoris, die assertio in libertatem, das interdictum de libero homine exhibendo und einige ähnliche Erscheinungen des Strafprocesses (VII). Die Entslehung der Popularklagen anlangend, so hält der Verfasser dafür, dass sie ,in erster Linie auf die im letzten Jahrhundert der Republik, besonders seit der Zeit der Gracchen, rasch zunehmende Durchtränkung des römischen Gemeinwesens mit demokratischen Elementen zurückzuführen" seien (VIII), wie umgekehrt in der späteren Kaiserzeit vermuthlich, in Folge der verändeıten Staatsverfassung ihre Zahl sich verringert hat", wäbrend freilich noch Justinian zwei ähnliche Rechtsmittel, die populare Klage auf Erfüllung von Vermächtnissen ad pias causas und diejenige auf Rückzahlung des Spielgewinnes, neu einführte (IX). 